\title{
Non-invasive DXA-derived bone structure assessment of acromegaly patients: a cross-sectional study
}

\author{
Martin Kužma', Peter Vaňuga², Ivana Ságová ${ }^{3}$ Dušan Pávai², Peter Jackuliak', Zdenko Killinger', Neil C Binkley4, \\ Renaud Winzenrieth ${ }^{5}$, Harry K Genant ${ }^{6}$ and Juraj Payer ${ }^{1}$
}

${ }^{1}$ Comenius University Faculty of Medicine, 5th Department of Internal Medicine, University Hospital Bratislava, Bratislava, Slovakia, ${ }^{2}$ National Institute of Endocrinology and Diabetology, Lubochna, Slovakia, ${ }^{3}$ Comenius University Jessenius Faculty of Medicine, 1 th Department of Internal Medicine, University Hospital Martin, Martin, Slovakia, ${ }^{4}$ Department of Medicine, University of Wisconsin, Madison, Wisconsin, USA, ${ }^{5}$ Galgo Medical, Barcelona, Spain, and ${ }^{6}$ University of California San Francisco, San Francisco, California, USA

Correspondence should be addressed to M Kužma

Email

kuzma@ru.unb.sk

\begin{abstract}
Introduction: Impaired bone microarchitecture is involved in vertebral fracture (VF) development among acromegaly patients.

Aim of the study: Comparison of DXA-derived bone parameters, areal BMD (aBMD), trabecular bone score (TBS) and 3D-SHAPER parameters in acromegaly patients with healthy controls.

Methods: This cross-sectional study evaluated acromegaly patients and a control group of healthy subjects. In all subjects, a single measurement of pituitary axis hormone levels, bone turnover markers, aBMD, (total hip (TH) and lumbar spine (LS)), TBS and 3D-SHAPER of the proximal femur region was performed. All subjects underwent DXA assessment of VF using the semiquantitative approach.

Results: One hundred six patients with acromegaly (mean age 56.6 years, BMI $30.2 \mathrm{~kg} / \mathrm{m}^{2}$ ) and 104 control subjects (mean age 54.06 years, $28.4 \mathrm{BMI} \mathrm{kg} / \mathrm{m}^{2}$ ) were included. After adjustment for weight, LS aBMD, TBS and TH trabecular volumetric BMD (VBMD) remained lower $(P=0.0048,<0.0001$ and $<0.0001$, respectively) while cortical thickness (Cth) at TH and neck remained thicker $(P=0.006)$ in acromegaly patients compared with controls. The best multivariate model (model 1) discriminating patients with and without acromegaly included TBS, TH trabecular vBMD and TH Cth parameters (all $P<0.05$ ). Twenty-two VFs (13 acromegaly subjects) were recognized. In these subjects after adjustment for age, FN aBMD, TH cortical SBMD and TH cortical VBMD remained significantly associated with the prevalent VF $(O R=2.69$ (1.07-6.78), 2.84 (1.24-6.51) and 2.38 (1.11-5.10) for neck aBMD, TH cortical SBMD and TH cortical vBMD respectively)). The AUCS were similar for each parameter in this model.

Conclusions: Acromegaly patients, regardless of VF presence, have lower trabecular bone quantitative parameters, but those with VFs had decreased cortical density.
\end{abstract}

\section{Introduction}

Acromegaly is caused by growth hormone hypersecretion $(\mathrm{GH})$, which affects the target tissues either directly or via insulin-like growth factor 1 (IGF-1). In bone, GH modulates osteoblast differentiation leading to increased (c) 2019 European Society of Endocrinology Printed in Great Britain bone formation. During childhood, GH has an important role in longitudinal bone growth; in adolescence, it helps to achieve peak bone mass and during adulthood GH affects bone remodeling $(1,2)$. GH and IGF-1 via 
renal 1- $\alpha$-hydroxylase stimulate the conversion of 25-OH-vitamin D to 1,25-dihydroxy-vitamin D, leading to calcium and phosphorus resorption in the gut and kidney (3). Thus, it is not surprising that some authors suggest positive effects of GH hypersecretion on bone (4, $5,6)$. However, several studies find increased prevalence of asymptomatic VFs in patients with acromegaly, regardless of bone mineral density (BMD) or disease activity $(7,8,9)$. In clinical practice, it is recommended to perform lateral spine scan imaging in acromegaly patients regardless of BMD to assess for potential vertebral fracture (VF) (10). Recognized risk factors for VF include hypogonadism, diabetes or previous VF.

Patients with acromegaly have elevated bone turnover markers $(11,12,13)$ but BMD as measured by dual-energy X-ray absorptiometry (DXA) may not adequately estimate fracture risk in patients with AG (1). Previously, it was thought that acromegaly patients have low BMD (13). More recently, it has been proven that $\mathrm{GH}$ hypersecretion is associated with normal or even higher BMD (1), which is partly explained by increased bone size and differing effects on cortical and trabecular bone (14, $15,16,17)$. Since DXA does not adequately explain the higher fracture risk observed in those with AG, it could be hypothesized that GH excess, whether directly or via hypogonadism, leads to bone microstructure changes $(16,17)$. Consistent with this, it has long been recognized that AG patients had increased bone width, increased cortical remodeling and normal or increased cortical bone mass but decreased trabecular bone mass (5). More recently, several quantitative computed tomography studies in acromegaly patients found poorer trabecular bone parameters, such as higher trabecular separation and decreased trabecular number and bone volume to tissue volume, in comparison to healthy controls (16). These conclusions are supported by studies with bone microidentation (18) and trabecular bone score (TBS) $(19,20)$. Finally, bone histomorphometric analysis in acromegaly patients with VFs showed impaired trabecular parameters (decreased trabecular thickness and increased trabecular separation), in comparison to healthy sexand age-matched adults and also increased cortical thickness and porosity among acromegaly subjects (15). In summary, existing evidence indicates that acromegaly patients have impaired trabecular bone microarchitecture leading to reduced bone strength and increased vertebral fracture risk.

Recently, another method for non-invasive bone structure assessment using DXA images called 3D-SHAPER was developed (21). 3D-SHAPER modeling provides a detailed proximal femur analysis including separate assessment of the cortical layer and trabecular macrostructure, which could potentially improve identification of those at elevated fracture risk $(21,22,23$, 24). This methodology has not been evaluated in patients with acromegaly. Therefore, the purpose of this crosssectional, case-control study was to evaluate which noninvasive and simply performed method; aBMD, LS TBS or proximal femur 3D-SHAPER analysis, could be of use to discriminate acromegalic patients at risk for $\mathrm{VF}$.

\section{Patients and methods}

\section{Patients}

This cross-sectional single-center study of AG patients was conducted from June 1, 2016 to November 30, 2017 at National Institute of Endocrinology and Diabetology, Lubochňa, Slovakia. The study protocol was reviewed and approved by National Institute of Endocrinology and Diabetology research boards. The regional medical ethics committee approved the study. All patients regularly followed at the endocrinology outpatient clinic who met inclusion criteria for any study group were included in the study; signed informed consent was obtained prior to the conduct of any study procedure.

The study included two groups:

(1) Acromegaly patients. Inclusion criteria were as follows:

- Diagnosis of acromegaly according to guidelines of Endocrine Society (25) regardless of gender, disease activity, gonadal or menopausal status within age 18-85 years at the time of study inclusion.

- No recognized cause of secondary osteoporosis other than acromegaly (e.g. malabsorption, rheumatoid arthritis, corticosteroid-induced osteoporosis, etc.).

- No history of osteoporosis treatment.

- No history of major trauma, which could lead to VF.

(2) Healthy age- and BMI-matched control subjects.

- Subjects who consecutively came to the outpatient office during the study time period and had no signs of acromegaly as assessed by normal IGF-1 values.

- No presence of primary or secondary osteoporosis (e.g. malabsorption, rheumatoid arthritis, corticosteroid-induced osteoporosis, etc.).

- No history of osteoporosis treatment. 
The acromegaly patients' group was subsequently analyzed according to gender, disease activity or presence of hypogonadism.

According to guidelines of the Endocrine Society (25) active disease was defined by:

(1) IGF-1 level above upper limit of normal reference range for age and (2) lack of suppression of GH to $<1 \mu \mathrm{g} / \mathrm{L}$ following documented hyperglycemia during an oral glucose load (No. 2 was not applied for patients treated with somatostatin receptor ligands or pegvisomant).

Otherwise, the disease was considered controlled disease, even when disease was maintained by ongoing treatment. Subjects were defined as hypogonadal if the total testosterone level was below $7.0 \mathrm{nmol} / \mathrm{L}$ in males, and absence of regular menstrual cycle in females.

All subjects were screened for impaired glucose tolerance (IGT) or overt diabetes mellitus (DM) according to American Diabetes Association guidelines (26), where fasting plasma glucose is increased; 5.6-6.9 and $>7 \mathrm{mmol} / \mathrm{L}$ for diagnosis IGT or DM is defined, respectively.

\section{Laboratory methods}

In all study subjects, serum levels of IGF-1, pituitary hormones, peripheral hormones and parameters of bone turnover were measured. Venous blood samples were taken after overnight fasting (minimum $8 \mathrm{~h}$ of fasting) between 07:00 and 08:30 h.

Total testosterone (TST), luteinizing hormone (LH), follicle-stimulating hormone (FSH), prolactin (PRL), thyroid-stimulating hormone (TSH) and free thyroxine (fT4) levels were measured by immunoassay using Siemens ADVIA Centaur systems. Adrenocorticotropic hormone (ACTH) and plasma cortisol levels were assessed with chemiluminescent immunometric assay (ECLIA) using IMMULITE 2000 assay. Plasma glucose and total calcium (Ca) levels were measured by Siemens Advia Chemistry.

IGF-1 levels were assessed by the IMMULITE assay, a solid phase, enzyme-labeled chemiluminescent immunometric assay (ECLIA) using IMMULITE 2500 assay (interassay variability CV: $2.4-4.7 \%$ ). Serum P1NP and CTx levels were assessed by ECLIA using a Roche Elecsys 1010/2010. P1NP values are expressed as $\mu \mathrm{g} / \mathrm{L}$ with interassay precision between 2.3 and 3.7\%. CTx values are expressed as $\mathrm{pg} / \mathrm{mL}$ with inter-assays precision of $1.6-4.7 \%$.

\section{BMD, TBS and 3D-SHAPER measures}

Each subject had BMD and TBS measurement performed \pm 7 days from blood sampling. BMD measurements at the
L1-4 spine, femoral neck (FN) and total hip (TH) were performed using DXA with a Hologic Horizon device with APEX software, version 13.3:7. Quality control was performed by daily calibration using the Hologic calibration phantom. The scans were acquired and analysis was performed by one technologist and the scan quality control was evaluated by experienced physicians (Dr Vaňuga and Dr Killinger). TBS was performed from LS DXA scans using TBS iNsight software (Medimaps SASU, Pessac, France) version 3.0.2.0.

\section{D-SHAPER measurement}

3D-SHAPER software (version 2.7, Galgo Medical S.L, Barcelona, Spain) was used to derive a 3D analysis from the hip DXA scans. Hip DXA scans were sent to Galgo Medical where the analysis was performed by a specialist (Dr Winzenrieth) without knowing the study cohort, that is acromegaly or control. Details of the 3D-SHAPER algorithm can be found in (21). Briefly, the method uses a 3D statistical shape and density model of the proximal femur built from a database of QCT scans of Caucasian men and women (21). This 3D statistical model is registered onto the hip DXA scan to obtain a 3D QCT-like patient-specific model of the proximal femur. The cortex is segmented subsequently by fitting a function to the density profile computed along the normal vector at each node of the proximal femur surface mesh (24). This fitting function depends on cortical thickness (Cth, in $\mathrm{mm}$ ), cortical volumetric BMD (cortical vBMD, in $\mathrm{mg} / \mathrm{cm}^{3}$ ), the location of the cortex, the density of surrounding tissues and the imaging blur. Once the cortex is segmented, the trabecular bone part is extracted and characterized as its volumetric BMD (trabecular vBMD, $\mathrm{g} / \mathrm{cm}^{3}$ ). A description of the process is presented in Fig. 1.

The cortical compartment is characterized by its thickness (Cth) and its volumetric BMD (cortical vBMD). These two parameters can vary either in similar fashion or in an opposite direction (e.g. with teriparatide treatment). To simplify characterization of the cortical compartment, the cortical surface BMD (cortical sBMD, in $\mathrm{mg} / \mathrm{cm}^{2}$ ) is computed, at each vertex of the femoral surface mesh, as the multiplication of the Cth (in $\mathrm{cm}$ ) by the cortical vBMD (in $\mathrm{g} / \mathrm{cm}^{3}$ ) observed along this thickness. Although new in the DXA field, cortical sBMD has been previously introduced and used to characterize the cortical compartment of the proximal femur from QCT acquisitions $(27,28,29)$. An increase (decrease) over time of Cth, cortical vBMD or both can induce an increase (decrease) of the cortical sBMD parameter. A thick but demineralized cortical bone 


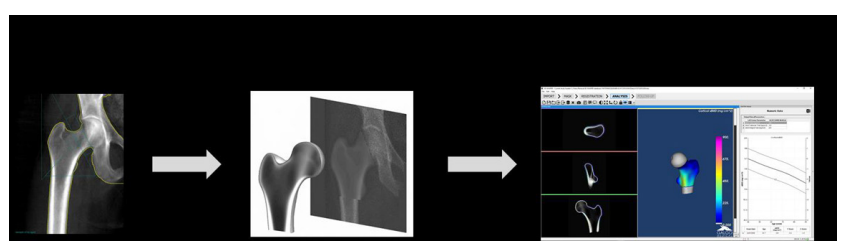

\section{Figure 1}

3D SHAPER workflow. From left to right: a femur DXA acquisition, the registration process and the results (QCT-like) of the DXA-based 3D modeling process. Basically, a population-based QCT model of the proximal femur is registered onto the DXA scan of the patient. At each step of the registration process, the $2 \mathrm{D}$ projection of this populationbased QCT model is compared with the DXA acquisition. As soon as the perfect match is obtained between projection and the DXA acquisition, the 3D patient-specific QCT-like model of the bone site is obtained. A full colour version of this figure is available at https://doi.org/10.1530/EJE-18-0881.

can have the same cortical sBMD value than a denser but thinner cortical bone. Furthermore, from a technical point of view, this parameter has the advantage to limit drawbacks effects linked to low resolution imaging such as the partial volume effect. Average values of the above-mentioned measurements are computed over the total hip ( $\mathrm{TH}$, union of the neck, the intertrochanteric and the shaft) region of interest as well as the neck, the intertrochanteric and the shaft regions.

\section{VF assessment}

Vertebral fracture assessment was performed using the lateral spine imaging IVA mode with a Hologic Horizon A densitometer (Hologic Inc.). Two lateral scans were performed in the supine position; one with single-energy and a second with dual-energy to ensure the best possible image quality. Each vertebra was assessed by co-author HKG using the Genant semiquantitative scoring method (30), grading as normal or mild, moderate or severe fracture. A subject was considered 'fractured' if a fracture was observed, regardless of if there were unreadable levels elsewhere.

\section{Statistical analysis}

Statistical analysis was performed using the statistics software SPSS version 19 (IBM SPSS Statistics, IBM Corporation). Continuous data were expressed as the mean \pm standard deviation (s.D.). Before statistical analysis, we have performed screening for outliers defined as values greater than +3 S.D.s or lower than -3 s.D.s for each variable, who were excluded from the study. The Shapiro-Francia test was used to test the normality of the distributions of studied parameter. Inter-group comparisons were performed using either a Student's t-tests or Mann-Whitney tests, depending upon the normality distribution of the studied parameter.

For bone-related measurements (DXA- and DXAbased 3D measurements), ANCOVA analysis was used to performed inter-group comparisons considering all other significant discriminant parameters as covariate. Univariate analyses and multivariate regressions (logistic regression using stepwise variable selection) were performed to investigate possible correlations between fracture risk and significant parameters. A measurement was entered into the model if its associated significance level was lower than 0.05 and excluded when $P$ was higher than 0.10. Odds ratios (OR) per standard deviation decreased or increased were calculated to evaluate the strength of association between independent variables and the presence of a prevalent fracture with and without covariate adjustments. Finally, an area under the receiveroperating characteristic curves (AUC) was used to assess the discriminating power of the significant parameters. AUC comparisons were carried out using the nonparametric DeLong test.

3D spatial distribution of changes (i.e. differences between subjects with and without acromegaly or with or without fracture) in the cortical bone (cortical sBMD, Cth and cortical vBMD) was computed. Mid-coronal slices were generated for each group and compared to display changes in vBMD. All inferential tests were two-tailed and Bonferroniadjusted threshold for statistical significance was set at 5\%.

\section{Results}

In total, 106 (38 males/68 females; average age of 56.3 years) acromegaly patients and 104 (16 males/88 females; average years of 54.1 years) control subjects were included in the study. Among acromegaly patients, 35 ( 33\%) had active disease, $16(\sim 15 \%)$ had hypogonadism and $13(\sim 12 \%)$ had at least one prevalent VF (Table 1). A number of $47(\sim 44 \%)$ acromegaly patients had IGT or DM.

\section{Treatment of acromegaly}

Within patients group, $82(\sim 77 \% ; 22$ active and 60 controlled) subjects were treated surgically, $22(\sim 20 \% ; 10$ active and 12 controlled) were treated with somatostatin 


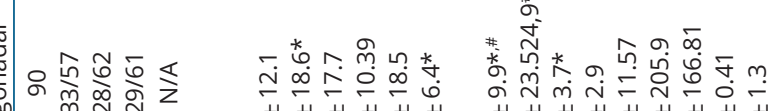

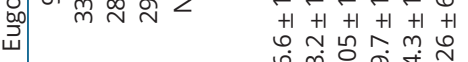

官

$+1+1+1+1+1+1+1+1+1$

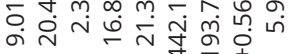

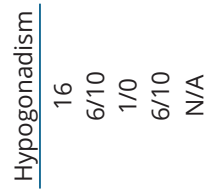

๖ำ $\stackrel{\infty}{\wedge} \stackrel{\infty}{\circ}$

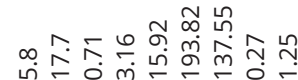

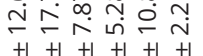

:

$+1+1+1+1+1+1+1+1$

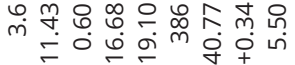

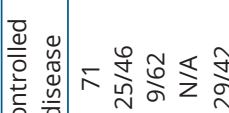

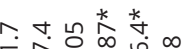

苞

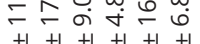

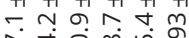

$\infty \sim \sim m \leftarrow N-\sigma^{-}$

$+1+1+1+1+1+1+1+1+1$

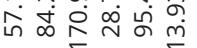

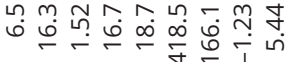

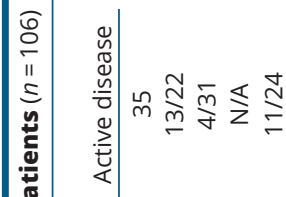

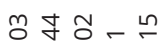

m் 잏 $\dot{m}$

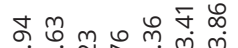

$+1+1+1+1+1+$

ทำ 워

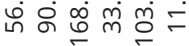

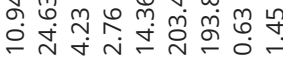

$+1+1+1+1+1+1+1+1+1$

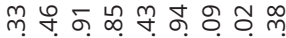

ำ ำ

$\mp \stackrel{\text { V }}{\sim} \dot{\sim} \underset{\mathcal{Y}}{\infty} \leftarrow$

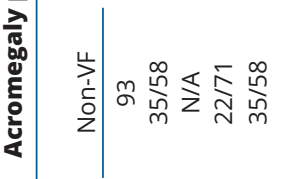

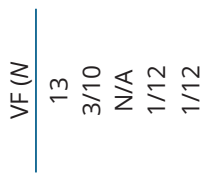

$-\leftarrow \sigma 06$

$n$
$+1+1+1+n$

ง

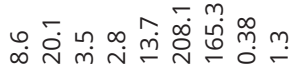

$+1+1+1+1+1+1+1$

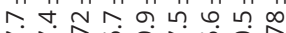

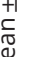

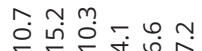

$+r^{+1}+\tau^{+1}+1+1+1$

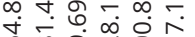

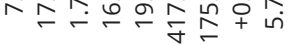

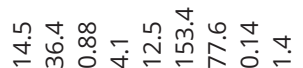

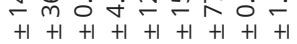



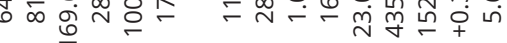

帮茾

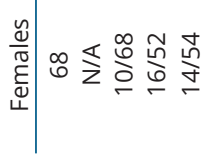

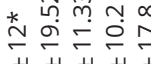

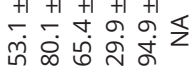

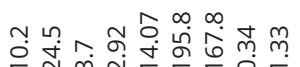
$+1+1+1+1+1+1+1+1$

จ. $\infty$ ก ก

产岗宁

ก. คั

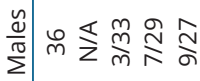

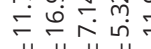

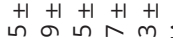

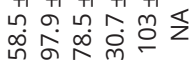

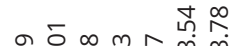

$\stackrel{\infty}{m} \underset{m}{m} \stackrel{\infty}{\sim}$

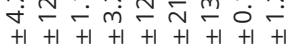

ำ

붕ํㅇ

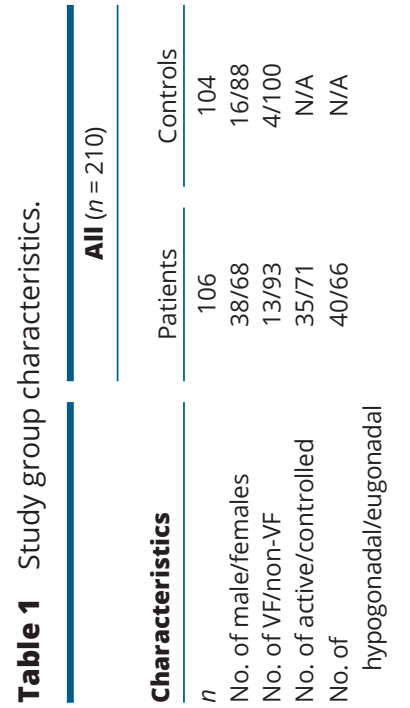

$\sim \stackrel{*}{6} m m \infty$

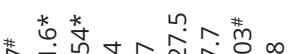

$+1+1+1+1+$

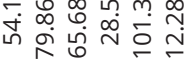

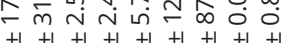

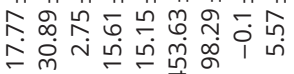

mִ $\stackrel{\infty}{\infty} \stackrel{\infty}{=} \infty \stackrel{m}{\varphi} \infty$

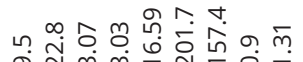

$+1+1+1+1+4$

+1 $+1+1+$

m율ำ

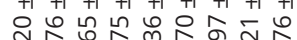

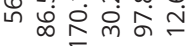

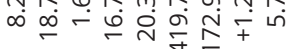
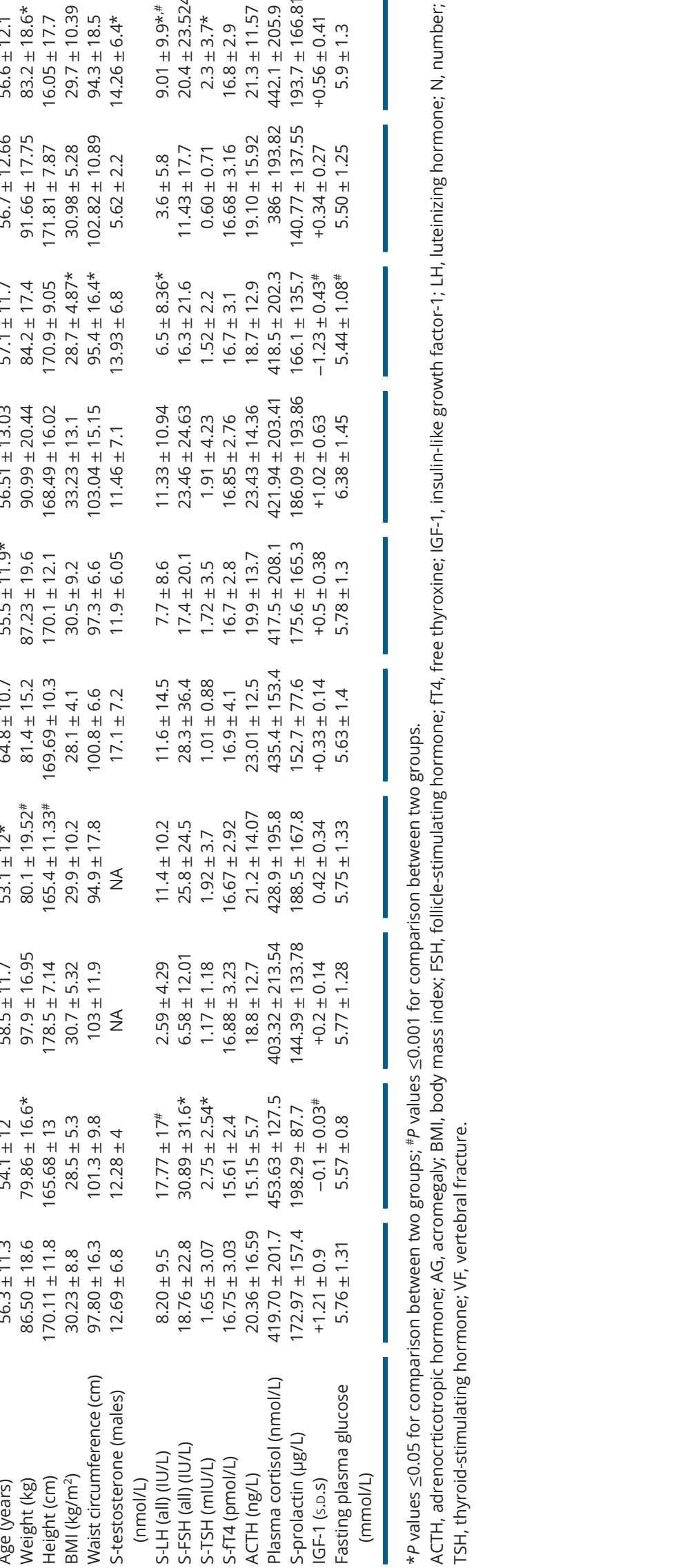
receptor ligands (SRLs) before surgery, 59 ( 55\%; 19 active and 40 controlled) were treated with SRLs after surgery with mean duration of 64.3 months, 22 ( 20\%; 12 active and 10 controlled) were treated with pegvisomant with mean duration of 43.5 month and 42 ( 39\%; 11 active and 31 controlled) were treated with radiotherapy.

\section{Comparison of acromegaly patients and healthy controls}

Age and BMI did not differ between acromegaly patients and control groups. Acromegaly patients were heavier and had higher IGF-1 and P1NP levels but lower LH, FSH, TSH and ACTH levels (all $P<0.05$ ).

Considering areal DXA bone parameters, acromegaly patients had significantly lower $(P<0.05)$ LS aBMD and TBS when compared to controls while no differences were observed for aBMD at the FN and $\mathrm{TH}$.

Using the 3D modeling approach, acromegaly patients had thicker Cth at both the FN $(P<0.05)$ and TH $(P \leq 0.001)$, but significantly lower trabecular vBMD at both sites (neck $(P<0.05)$ and TH $(P<0.001))$.

After adjustment for weight, LS aBMD, TBS and TH trabecular vBMD remained significantly lower $(P=0.0048$, $<0.0001$ and $<0.0001$ respectively) in acromegaly patients compared with controls. In contrast, $\mathrm{Cth}$ (at TH and neck) remained significantly thicker $(P=0.006)$ in acromegaly patients than in controls (Fig. 2).

Considering bone parameters only (2D and 3D), the best multivariate model (model 1) discriminating patients with and without acromegaly included TBS, TH trabecular vBMD and TH Cth parameters while aBMD LS and weight were excluded from the model (all $P>0.1$ ). Model 1 (Fig. 3) was associated with the presence of acromegaly as reported by an OR per s.D. decrease of 4.85 (3.09-7.62) and an AUC of 0.845 (0.787-0.903). AUC of model 1 was significantly greater than AUCs of each of the three co-factors included in this model (all $P<0.01$ ).

\section{Acromegaly males vs acromegaly females}

Among acromegaly patients, 60\% were women. Male patients were older, taller and heavier when compared to women (all $P<0.05$ ). Considering bone measurements, males had higher aBMD at both spine $(P=0.042)$ and femur $(P<0.0001)$ as well as thicker femoral cortex $(P<0.0001)$ and higher cortical sBMD $(P=0.0004)$. Multivariate analysis demonstrated that height parameter was the best discriminator between males and females, and all other parameters were excluded from the analysis $(P>0.1)$.
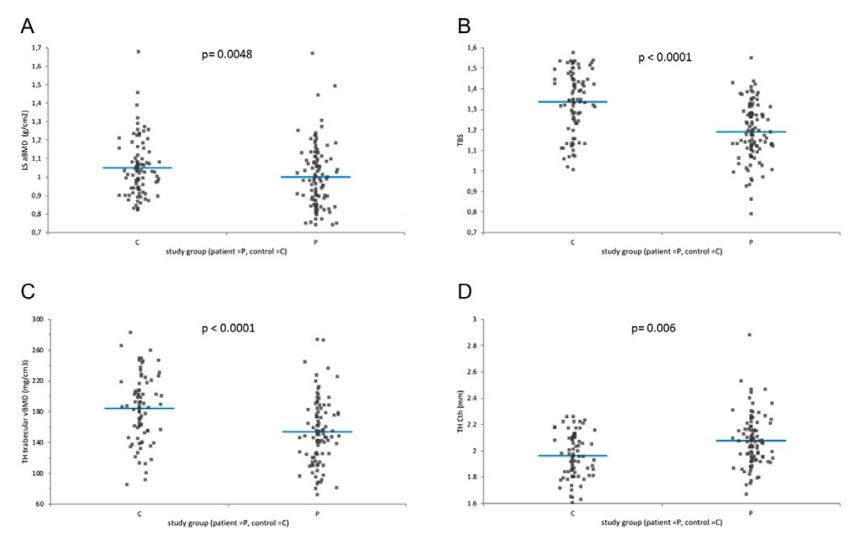

\section{Figure 2}

After adjustment for weight, (A) aBMD LS $(P=0.0048)$, (B) TBS $(P<0.0001)$ and $(C)$ trabecular VBMD TH $(P<0.0001)$ remained lower in acromegaly patients compared with controls while Cth (at (D) TH and neck) remained thicker $(P=0.006)$. A full colour version of this figure is available at https://doi. org/10.1530/EJE-18-0881.

\section{Active vs controlled disease}

Acromegaly patients with active disease had greater BMI, waist circumference, LH, IGF-1, FPG and P1NP in comparison to patients with controlled disease. No difference in terms of $2 \mathrm{D}$ or $3 \mathrm{D}$ bone measurements was observed. The best measurement discriminating

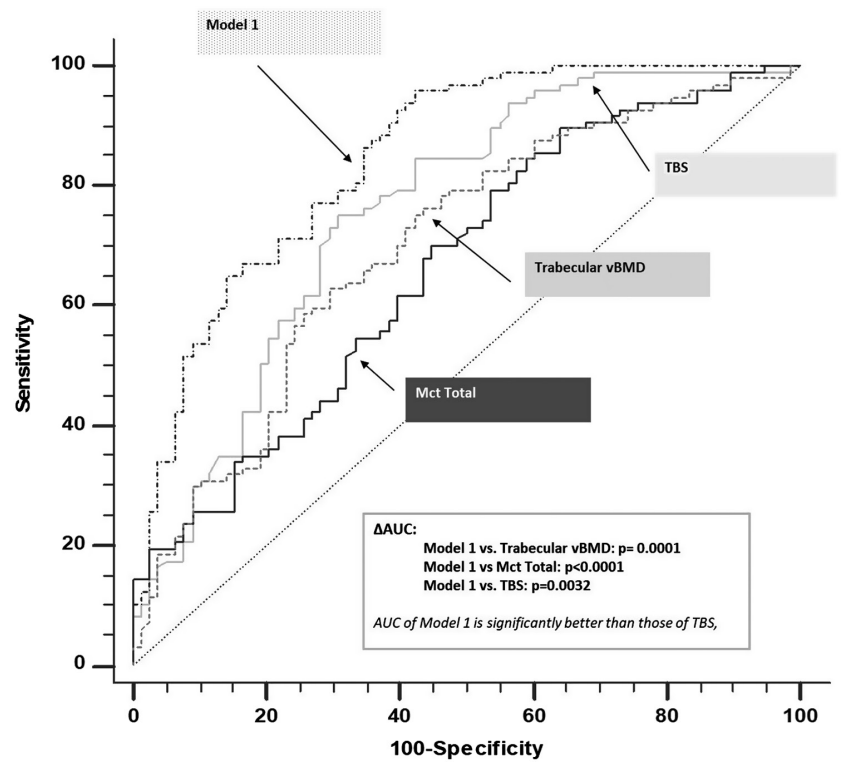

Figure 3

AUC of model 1 (TBS, TH trabecular VBMD and TH Cth) is better than those of TBS, TH trabecular VBMD and TH CTh alone. 


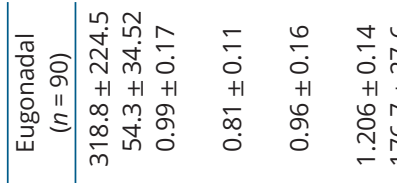

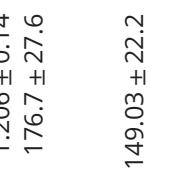

$\therefore \approx 0$

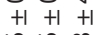

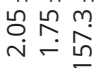

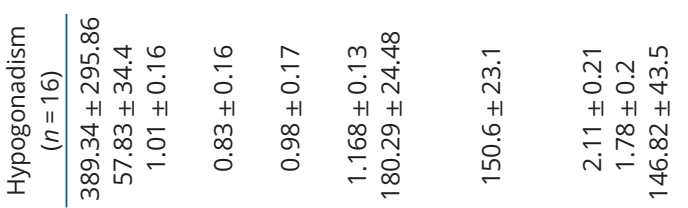

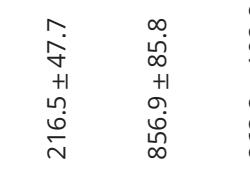

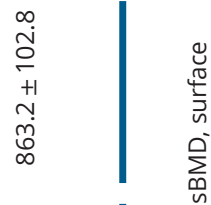

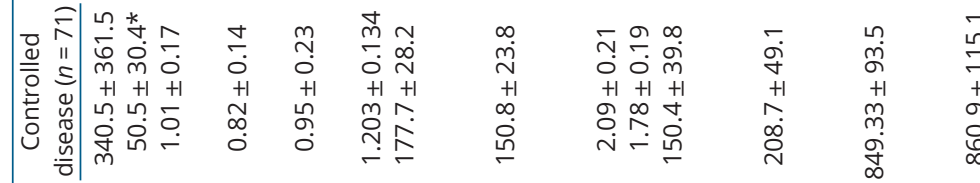

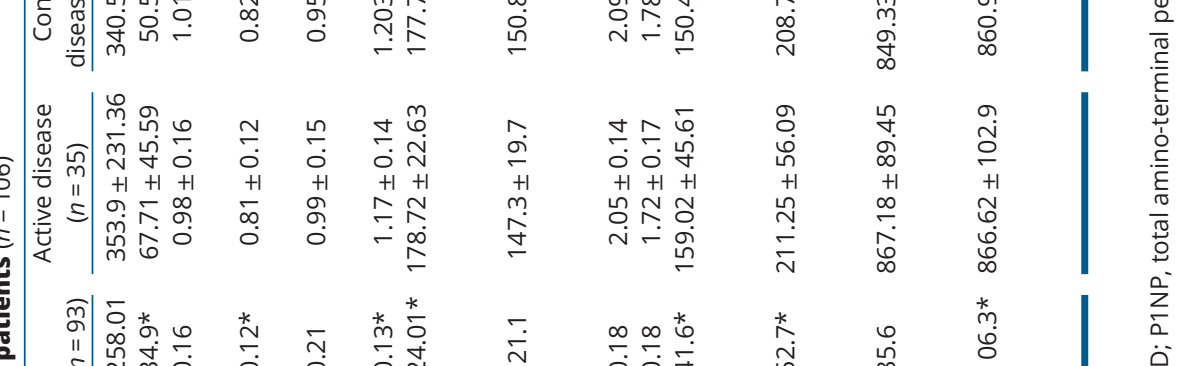

$\infty \infty \stackrel{*}{*} \stackrel{*}{\wedge} \quad \stackrel{*}{n}$

ரேं तु

突突

完|

$+1$

$+1$

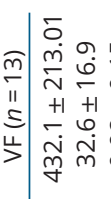

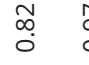

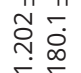

$\stackrel{+}{m}$

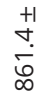

+1
+
$\infty$
$\infty$
$\infty$

ำำ ํํㅇ

$+1+1$

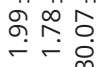

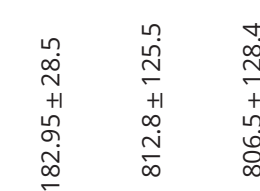

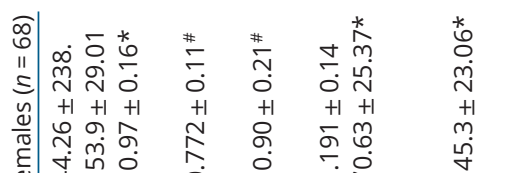

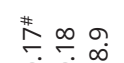

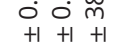

i.

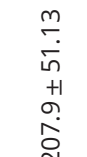

$r$
$\infty$
$\infty$
+1
+
$\dot{J}$
$\infty$

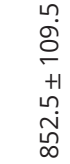

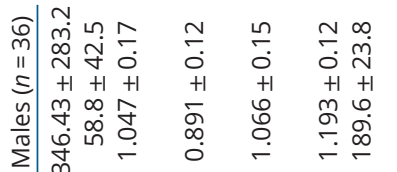

등ำำ

$+1+1$

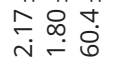

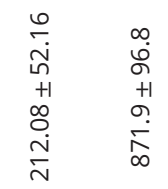

$\underset{\substack{\infty \\ \leftarrow \\+1 \\ \infty \\ \infty \\ \infty \\ \infty \\ \infty}}{\infty}$

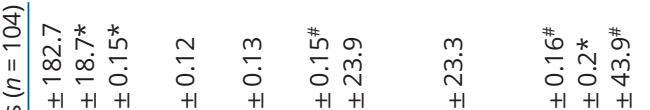

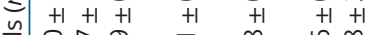

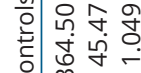

so.

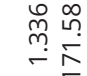

운 윰

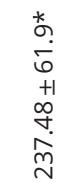

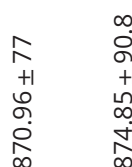

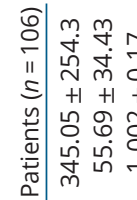

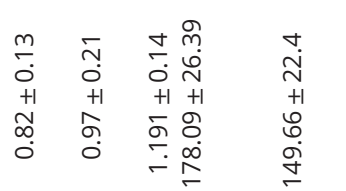

$\stackrel{0}{0} \div \stackrel{\infty}{\dot{0}}$

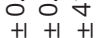

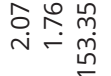

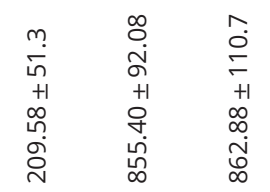

ஸे

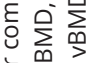

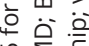


individuals with active disease and those with controlled disease was IGF-1 level. Acromegaly patients with active disease had significantly higher IGF-1 level (408 vs $158 \mathrm{ng} / \mathrm{mL}, P<0.001)$.

\section{Hypogonadal vs eugonadal AG subjects}

Among acromegaly patients, hypogonadal patients were heavier than eugonadal patients $(P=0.025)$ and had lower level of LH and FSH (all $P<0.01$ ). No differences were observed in terms of age, BMI, IGF-1, CTx and P1NP levels.

After adjustment for weight, trabecular vBMD, LH and FSH remained significantly lower $(P=0.048, P=0.0003$ and $P=0.0008$ respectively) in hypogonadal patients while other 2D or 3D measurements were not (all $P>0.10$ ). LH, FSH and trabecular vBMD remained significantly lower in hypogonadal patients $(P=0.011)$ and associated with the presence of hypogonadism as expressed by an OR per S.D. decrease of 20.9 (3.95-110.17), 6.49 (2.15-19.5) and 1.76 (1.07-2.88) respectively.

\section{Acromegaly subjects with vs without VFs.}

Patients with prevalent VF were older $(P=0.017)$ than those without while the two groups were similar in terms weight, height or BMI (Table 1). Both 2D and 3D measurements as well as TBS were significantly lower in subjects with VF (all $P<0.05$ ) considering either the total femur or the spine regions (Table 2).

In 13 acromegaly patients, a total number of 22 VFs was identified. Four control group subjects had VFs

Table 3 Characteristics of subjects with VFs.

\begin{tabular}{|c|c|c|}
\hline & AG subjects & Control group \\
\hline Number of VFs & 22 & 4 \\
\hline \multicolumn{3}{|l|}{ Severity of VFs } \\
\hline Mild & 10 & 4 \\
\hline Moderate & 12 & 0 \\
\hline Severe & 0 & 0 \\
\hline Number of subjects with $\geq 1 \mathrm{VF}$ & 13 & 4 \\
\hline Number of subjects with multiple VFs & 6 & 0 \\
\hline Disease activity & & NA \\
\hline Active & 1 & \\
\hline Controlled & 12 & \\
\hline \multicolumn{3}{|l|}{ Gender } \\
\hline Males & 3 & 2 \\
\hline Females & 10 & 2 \\
\hline \multicolumn{3}{|l|}{ Hypogonadism } \\
\hline Yes & 1 & 0 \\
\hline No & 12 & 0 \\
\hline \multicolumn{3}{|l|}{ Diabetes mellitus or IGT } \\
\hline Yes & 7 & 0 \\
\hline No & 6 & 0 \\
\hline
\end{tabular}

identified. Characteristics of subjects with VFs in Table 3. After adjustment for age, neck aBMD, TH cortical sBMD and TH cortical vBMD remained significantly associated with the prevalent VF $(\mathrm{OR}=2.69$ (1.07-6.78), 2.84 (1.246.51) and 2.38 (1.11-5.10) for neck aBMD, TH cortical sBMD and TH cortical vBMD respectively, Fig. 4A, B, C and $\mathrm{D}$ ) while aBMD at spine, trabecular vBMD TF, TBS and Cth were not associated with the fracture. The AUC analysis showed slight superiority of TH cortical sBMD. The AUC was 0.754 (0.598-0.911), 0.793 (0.603-0.983) and 0.765 (0.579-0.950) for neck aBMD, Th cortical sBMD and TH cortical vBMD respectively (Fig. 4D). Average differences in both types of vBMD (trabecular and cortical) between acromegaly subjects with and without VF are shown in Fig. 5.

\section{Discussion}

Acromegaly is associated with increased VF prevalence (1), despite normal or even elevated BMD. The mechanism of these fractures is not fully understood. In this study, we report lower LS aBMD, TBS and TH vBMD, but higher hip cortical bone parameters in acromegaly patients. This impairment of trabecular structure could potentially explain the higher VF risk in AG patients. Nevertheless, this study finds in 13 acromegaly patients with VFs,
A

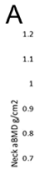

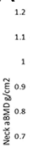
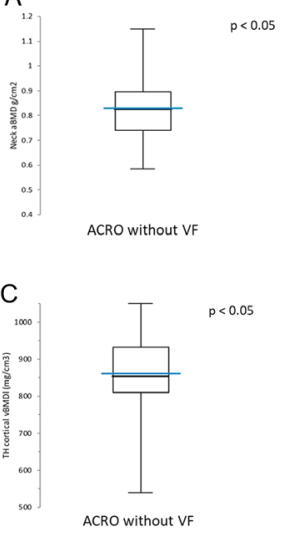

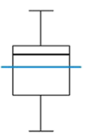

ACRO with VF
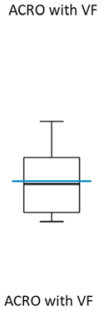

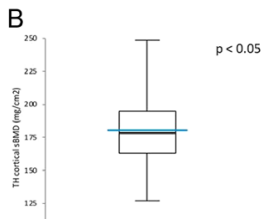

ACRO without $Y F$

D

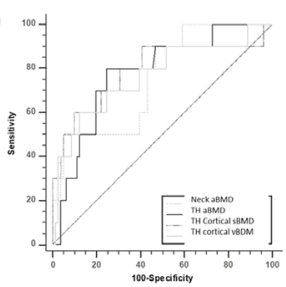

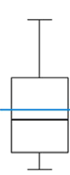

ACRO with VF

\section{Figure 4}

Differences between acromegaly subjects with vs without vertebral fracture. After adjustment for age, (A) neck aBMD, (B) TH cortical SBMD and (C) TH cortical VBMD remained significantly associated with the prevalent VF. The AUC analysis showed slight superiority of TH cortical SBMD (D). A full colour version of this figure is available at https://doi.org/10.1530/ EJE-18-0881. 


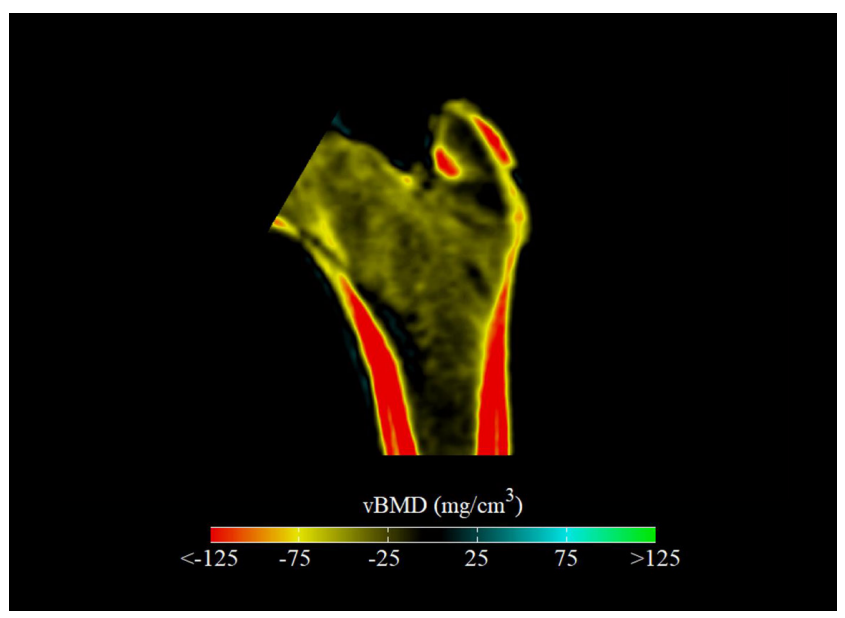

\section{Figure 5}

Average differences in both types of VBMD (trabecular and cortical) between acromegaly subjects with and without VF. Differences are shown in the mid-coronal plane. Red-yellow (respectively blue-green) colors indicate regions where vBMD is on average lower (respectively higher) for acromegaly subjects compared to controls.

decreased cortical vBMD and sBMD at hip region. This study advances acromegaly knowledge by reporting TBS, a bone texture surrogate derived from DXA scan of LS and 3D-SHAPER measures non-invasive 3D modeling of bone structure assessment from proximal femur DXA scans. Both TBS and 3D-SHAPER could be easily obtained in clinical settings. After multivariate analysis of $2 \mathrm{D}$ and 3D bone measures, only TBS, TH trabecular vBMD and TH Cth remained significantly different in AG patients in comparison to healthy controls. Additionally, sensitivity and specificity was best when all three parameters were together in one model in comparison to each parameter alone. This is in agreement to previous studies done with 'gold standard' methods for bone microarchitecture assessment, for example, bone histomorphometry and HR-pQCT $(15,16,17,19,20)$. Additionally, analysis of gonadal status revealed lower trabecular vBMD at the proximal femur, but these results are impacted by the small number of hypogonadal (treatment naïve) acromegaly patients. It is plausible that the impaired trabecular architecture observed in this acromegaly cohort is the result of hypogonadism, which is known to cause trabecular microarchitectural degeneration in both men and women. However, as the time from disease onset to diagnosis and treatment is long (years), the impact of untreated disease with or without concomitant hypogonadism could also affect the bone.
In a prior study of high resolution cone-beam tomography in acromegaly patients with radiological VFs lower trabecular volume, greater trabecular separation and higher cortical porosity were observed in comparison to non-fractured patients (16). According to these results, it is likely that not only the trabecular compartment but also the cortical compartment with decreased volumetric and surface density plays a role in fracture development among acromegaly patients. This was most recently supported by bone histomorphometry data (15) which showed reduced trabecular bone parameters and increased porosity in acromegaly subjects with VFs. We acknowledge that in the present study those with VF were older and female gender was more prevalent, which leads to doubts that we can directly link fracture status with acromegaly presence but more with a combination of age, gender, acromegaly and gonadal status. However, the number of VFs does not allow us to compare subsets with regard to age, gender and gonadal status. Further study is needed to clarify this relationship.

The study has several strengths including a large number of well-defined subjects for a rare disease and three different methods easily obtained from DXA technology, For example, aBMD, TBS and 3D SHAPER parameters. Additionally, VF identification is challenging, especially when fractures are mild and involve the upper thoracic vertebrae (31); we minimized this confounder by readings performed by HKG, a recognized expert in the field. Previously, the use of DXA assessment of VFs showed reasonable agreement with morphometry performed on standard X-ray and is recommended as alternative to conventional radiography (32). Limitations are crosssectional study design, which did not allow specify the time of VF and inclusion of postmenopausal females. A different proportion of both genders were included in the acromegaly and control group; however, it has no impact on study results.

To conclude, this study shows that acromegaly patients after multivariate analysis have lower TBS, trabecular vBMD and higher Cth, as measured by a novel method 3D-SHAPER, in comparison to healthy controls. It is likely that hypogonadism contributes to alteration of trabecular bone, as it was observed by decreased trabecular vBMD at the hip region. Additionally, patients with acromegaly had prevalent asymptomatic VFs that are associated with decreased FN aBMD, TH cortical vBMD and surface BMD. There was no association between bone measures and gender or disease activity.

In conclusion, this study indicates that, in addition to trabecular bone alterations known from previous studies, 
cortical bone also likely plays an important role among acromegaly subjects with prevalent VFs. However, it is likely that cofounding factors such as age, gender and gonadal status contribute to bone alterations leading to predisposition to fractures among acromegaly subjects. Observations from this study, supported by the findings of previous studies, allow us to conclude that in the routine practice selected parameters from 3D-SHAPER, could discriminate acromegaly patients at risk for VFs where there is no access to HR-pQCT. Further longitudinal studies are needed to explore this issue.

\section{Declaration of interest}

Renaud Winzenrieth is employed by Galgo Medical. The other authors have nothing to disclose.

\section{Funding}

This research did not receive any specific grant from any funding agency in the public, commercial or not-for-profit sector.

\section{References}

1 Mazziotti G, Biagioli E, Maffezzoni F, Spinello M, Serra V, Maroldi R, Floriani I \& Giustina A. Bone turnover, bone mineral density, and fracture risk in acromegaly: a meta-analysis. Journal of Clinical Endocrinology and Metabolism 2015100 384-394. (https://doi. org/10.1210/jc.2014-2937)

2 Giustina A, Mazziotti G \& Canalis E. Growth hormone, insulin-like growth factors, and the skeleton. Endocrine Reviews 200829 535-559. (https://doi.org/10.1210/er.2007-0036)

3 Kamenicky P, Mazziotti G, Lombes M, Giustina A \& Chanson P. Growth hormone, insulin-like growth factor-1, and the kidney: pathophysiological and clinical implications. Endocrine Reviews 2014 35 234-281. (https://doi.org/10.1210/er.2013-1071)

4 Vestergaard P \& Mosekilde L. Fracture risk is decreased in acromegaly - a potential beneficial effect of growth hormone. Osteoporosis International 200415 155-159. (https://doi.org/10.1007/s00198-003-1531-z)

5 Riggs BL, Randall RV, Wahner HW, Jowsey J, Kelly PJ \& Singh M. The nature of the metabolic bone disorder in acromegaly. Journal of Clinical Endocrinology and Metabolism 197234 911-918. (https://doi. org/10.1210/jcem-34-6-911)

6 Kayath MJ \& Vieira JG. Osteopenia occurs in a minority of patients with acromegaly and is predominant in the spine. Osteoporosis International 19977 226-230. (https://doi.org/10.1007/ BF01622293)

7 Bonadonna S, Mazziotti G, Nuzzo M, Bianchi A, Fusco A, De Marinis L \& Giustina A. Increased prevalence of radiological spinal deformities in active acromegaly: a cross-sectional study in postmenopausal women. Journal of Bone and Mineral Research 2005 20 1837-1844. (https://doi.org/10.1359/JBMR.050603)

8 Mazziotti G, Bianchi A, Porcelli T, Mormando M, Maffezzoni F, Cristiano A, Giampietro A, De Marinis L \& Giustina A. Vertebral fractures in patients with acromegaly: a 3-year prospective study. Journal of Clinical Endocrinology and Metabolism 201398 3402-3410. (https://doi.org/10.1210/jc.2013-1460)

9 Claessen KM, Kroon HM, Pereira AM, Appelman-Dijkstra NM, Verstegen MJ, Kloppenburg M, Hamdy NA \& Biermasz NR.
Progression of vertebral fractures despite long-term biochemical control of acromegaly: a prospective follow-up study. Journal of Clinical Endocrinology and Metabolism 201398 4808-4815. (https:// doi.org/10.1210/jc.2013-2695)

10 Melmed S, Casanueva FF, Klibanski A, Bronstein MD, Chanson P, Lamberts SW, Strasburger CJ, Wass JA \& Giustina A. A consensus on the diagnosis and treatment of acromegaly complications. Pituitary 201316 294-302. (https://doi.org/10.1007/s11102-012-0420-x)

11 Ueland T, Bollerslev J, Godang K, Muller F, Froland SS \& Aukrust P. Increased serum osteoprotegerin in disorders characterized by persistent immune activation or glucocorticoid excess - possible role in bone homeostasis. European Journal of Endocrinology 2001145 685-690. (https://doi.org/10.1530/eje.0.1450685)

12 Stepan J, Marek J, Havranek T, Dolezal V \& Pacovsky V. Bone isoenzyme of serum alkaline phosphatase in acromegaly. Clinica Chimica Acta 197993 355-363. (https://doi.org/10.1016/00098981(79)90285-7)

13 Scillitani A, Chiodini I, Carnevale V, Giannatempo GM, Frusciante V, Villella M, Pileri M, Guglielmi G, Di Giorgio A, Modoni S et al. Skeletal involvement in female acromegalic subjects: the effects of growth hormone excess in amenorrheal and menstruating patients. Journal of Bone and Mineral Research 199712 1729-1736. (https://doi. org/10.1359/jbmr.1997.12.10.1729)

14 Diamond T, Nery L \& Posen S. Spinal and peripheral bone mineral densities in acromegaly: the effects of excess growth hormone and hypogonadism. Annals of Internal Medicine 1989111 567-573. (https://doi.org/10.7326/0003-4819-111-7-567)

15 Dalle Carbonare L, Micheletti V, Cosaro E, Valenti MT, Mottes M, Francia G \& Davi MV. Bone histomorphometry in acromegaly patients with fragility vertebral fractures. Pituitary 201821 56-64. (https://doi.org/10.1007/s11102-017-0847-1)

16 Maffezzoni F, Maddalo M, Frara S, Mezzone M, Zorza I, Baruffaldi F, Doglietto F, Mazziotti G, Maroldi R \& Giustina A. High-resolutioncone beam tomography analysis of bone microarchitecture in patients with acromegaly and radiological vertebral fractures. Endocrine 201654 532-542. (https://doi.org/10.1007/s12020-0161078-3)

17 Madeira M, Neto LV, de Paula Paranhos Neto F, Barbosa Lima IC, Carvalho de Mendonca LM, Gadelha MR \& Fleiuss de Farias ML. Acromegaly has a negative influence on trabecular bone, but not on cortical bone, as assessed by high-resolution peripheral quantitative computed tomography. Journal of Clinical Endocrinology and Metabolism 201398 1734-1741. (https://doi.org/10.1210/jc.20124073)

18 Malgo F, Hamdy NAT, Papapoulos SE \& Appelman-Dijkstra NM. Bone material strength index as measured by impact microindentation is low in patients with fractures irrespective of fracture site. Osteoporosis International 201728 2433-2437. (https://doi.org/10.1007/s00198017-4054-8)

19 Godang K, Olarescu NC, Bollerslev J \& Heck A. Treatment of acromegaly increases BMD but reduces trabecular bone score: a longitudinal study. European Journal of Endocrinology $2016 \mathbf{1 7 5}$ 155-164. (https://doi.org/10.1530/EJE-16-0340)

20 Hong AR, Kim JH, Kim SW, Kim SY \& Shin CS. Trabecular bone score as a skeletal fragility index in acromegaly patients. Osteoporosis International 201627 1123-1129. (https://doi.org/10.1007/s00198015-3344-2)

21 Humbert L, Martelli Y, Fonolla R, Steghofer M, Di Gregorio S, Malouf J, Romera J \& Barquero LM. 3D-DXA: assessing the femoral shape, the trabecular macrostructure and the cortex in 3D from DXA images. IEEE Transactions on Medical Imaging 201736 27-39. (https:// doi.org/10.1109/TMI.2016.2593346)

22 Freitas L, Amorim T, Humbert L, Fonolla R, Flouris AD, Metsios GS, Jamurtas AZ \& Koutedakis Y. Cortical and trabecular bone analysis of professional dancers using 3D-DXA: a case-control study. Journal of 
Sports Sciences 2018 Epub. (https://doi.org/10.1080/02640414.2018. 1483178)

23 Gifre L, Humbert L, Muxi A, Del Rio L, Vidal J, Portell E, Monegal A, Guanabens N \& Peris P. Analysis of the evolution of cortical and trabecular bone compartments in the proximal femur after spinal cord injury by 3D-DXA. Osteoporosis International 201829 201-209. (https://doi.org/10.1007/s00198-017-4268-9)

24 Humbert L, Hazrati Marangalou J, Del Rio Barquero LM, van Lenthe GH \& van Rietbergen B. Technical note: cortical thickness and density estimation from clinical CT using a prior thicknessdensity relationship. Medical Physics 201643 1945. (https://doi. org/10.1118/1.4944501)

25 Katznelson L, Laws ER, Jr., Melmed S, Molitch ME, Murad MH, Utz A, Wass JA \& Endocrine Society. Acromegaly: an Endocrine Society Clinical Practice Guideline. Journal of Clinical Endocrinology and Metabolism 201499 3933-3951. (https://doi.org/10.1210/jc.2014-2700)

26 Chamberlain JJ, Rhinehart AS, Shaefer CF Jr \& Neuman A. Diagnosis and management of diabetes: synopsis of the 2016 American Diabetes Association standards of medical care in diabetes. Annals of Internal Medicine 2016164 542-552. (https://doi.org/10.7326/M153016)

27 Treece GM \& Gee AH. Independent measurement of femoral cortical thickness and cortical bone density using clinical CT. Medical Image Analysis 201520 249-264. (https://doi.org/10.1016/j. media.2014.11.012)
28 Poole KE, Treece GM, Gee AH, Brown JP, McClung MR, Wang A \& Libanati C. Denosumab rapidly increases cortical bone in key locations of the femur: a 3D bone mapping study in women with osteoporosis. Journal of Bone and Mineral Research 201530 46-54. (https://doi.org/10.1002/jbmr.2325)

29 Nicks KM, Amin S, Melton LJ, 3rd, Atkinson EJ, McCready LK, Riggs BL, Engelke K \& Khosla S. Three-dimensional structural analysis of the proximal femur in an age-stratified sample of women. Bone 201355 179-188. (https://doi.org/10.1016/j.bone.2013.02.009)

30 Genant HK, Jergas M, Palermo L, Nevitt M, Valentin RS, Black D \& Cummings SR. Comparison of semiquantitative visual and quantitative morphometric assessment of prevalent and incident vertebral fractures in osteoporosis the Study of Osteoporotic Fractures Research Group. Journal of Bone and Mineral Research 1996 11 984-996. (https://doi.org/10.1002/jbmr.5650110716)

31 Rea JA, Li J, Blake GM, Steiger P, Genant HK \& Fogelman I. Visual assessment of vertebral deformity by X-ray absorptiometry: a highly predictive method to exclude vertebral deformity. Osteoporosis International 200011 660-668. (https://doi.org/10.1007/ s001980070063)

32 Hoyer-Kuhn H, Knoop K, Semler O, Kuhr K, Hellmich M, Schoenau E \& Koerber F. Comparison of DXA scans and conventional X-rays for spine morphometry and bone age determination in children. Journal of Clinical Densitometry 201619 208-215. (https://doi.org/10.1016/j. jocd.2015.04.006)

Received 1 November 2018

Revised version received 17 December 2018

Accepted 19 December 2018 